\title{
Early Adjacent Vertebral Fractures after Balloon Kyphoplasty for Osteoporotic Vertebral Compression Fractures
}

\author{
Bong-Seong Ko, Kyu-Jung Cho, Jae-Woo Park \\ Department of Orthopedic Surgery, Inha University School of Medicine, Incheon, Korea
}

\begin{abstract}
Study Design: Retrospective cohort study.
Purpose: To evaluate the incidence and risk factors for early adjacent vertebral fractures following balloon kyphoplasty (KP).

Overview of Literature: $\mathrm{KP}$ is a safe and effective treatment for pain alleviation in patients with osteoporotic vertebral compression fractures (OVCF). However, some studies have reported that the risk of newly developed fractures increases at the adjacent vertebra after KP.

Methods: Total 123 consecutive patients with painful OVCF who underwent KP were enrolled from January 2009 to June 2016. Early adjacent vertebral fractures were defined as new fractures that had developed within 3 months after surgery. Sex, age, body mass index (BMI), bone mineral density (BMD), vertebral height, kyphotic angle, Visual Analog Scale score, cement amount, and leakage were evaluated as risk factors for adjacent vertebral fractures. Only cement leakage into the disc space was included in this study. Results: Early adjacent vertebral fractures were identified in $20(16.2 \%)$ of the 123 patients. The mean time to diagnosis of fractures was $1.7 \pm 0.7$ months after KP. The average patient age was $78.0 \pm 0.7$ years, average BMl was $23.06 \pm 3.83 \mathrm{~kg} / \mathrm{m}^{2}$, and mean BMD was $-3.61 \pm 1.22 \mathrm{~g} / \mathrm{m}^{2}$. Cement leakage was present in 16 patients, and fractures developed in $11(68.7 \%)$. In contrast, fractures developed in nine patients (8.2\%) without cement leakage. There were no significant differences in terms of age, BMI, BMD, kyphotic angle, or vertebral body height ratio between the fracture and control groups.

Conclusions: Cement leakage into the disc increased the risk of early adjacent vertebral fractures after balloon KP.
\end{abstract}

Keywords: Osteoporotic vertebral compression fracture; Adjacent vertebral fracture; Balloon kyphoplasty; Cement leakage

\section{Introduction}

Osteoporotic vertebral compression fractures (OVCF) comprise a major health problem that decrease the quality of life and increase back pain [1-5]. Balloon kyphoplasty $(\mathrm{KP})$ is a procedure wherein a deflated balloon is inserted into the vertebral body through the pedicle and then inflated to restore the height of a collapsed vertebral body. The balloon is then deflated and withdrawn, and the remaining cavity is filled with polymethyl methacrylate (PMMA) or a bone substitute cement [6,7].

$\mathrm{KP}$ is a rapid, less invasive, effective method for reducing pain in symptomatic vertebral compression fractures [8]. Although KP has been reported to be associated with

Received Aug 31, 2018; Revised Sep 3, 2018; Accepted Sep 7, 2018

Corresponding author: Kyu-Jung Cho

Department of Orthopedic Surgery, Inha University Hospital, 27 Inhang-ro, Jung-gu, Incheon 22332, Korea

Tel: +82-32-890-3043, Fax: +82-32-890-3047, E-mail: chokj@inha.ac.kr 
fewer complications than vertebroplasty (VP), it causes complications such as cement leakage, spinal cord injury, pulmonary embolus, and adjacent vertebral fractures $[9,10]$. In particular, early adjacent vertebral fractures that occur within few months after surgery do not reduce back pain and exert negative effects on surgical outcomes. Spross et al. [11] demonstrated that adjacent vertebral fractures were present in $9.9 \%$ of 375 patients after KP who were followed up for an average of 3.6 months. Risk factors for adjacent fractures included preoperative segmental kyphosis of $>30^{\circ}$ or comorbidities such as rheumatoid arthritis and cardiovascular disease [11]. Lin et al. [12] reported new fractures in 14 of 38 patients treated with VP, 10 with new fractures associated with cement leakage into the disk, and four with new fractures not associated with cement leakage.

There are still controversy regarding risk factors for adjacent vertebral fractures after KP. This study aimed to assess the incidence and risk factors for early adjacent vertebral fractures related to KP in patients with OVCF.

\section{Materials and Methods}

\section{Subjects}

Between January 2009 and June 2016, a total of 123 consecutive patients (143 vertebrae) with painful OVCF who underwent KP were enrolled. Each patient was followed up for at least 6 months. The study population comprised 92 women and 31 men. Vertebral compression fractures were confirmed using computed tomography (CT) and magnetic resonance imaging (MRI). If needed, vertebral compression fractures were further confirmed by evaluating bony edema in fat-suppressed sequences using MRI.

This study included patients with OVCF, excluding pathologic fractures related to malignancy. Cement leakage developing via cortical defects was found in the disc space, in the epidural space behind the posterior wall, and anterior to the body. Cement was also found in the external vertebral venous plexus, which caused pulmonary embolism. This study included only patients with cement leakage into the disc space that might have been associated with adjacent segment fractures. Early adjacent vertebral fractures were defined as new fractures that had developed within 3 months after surgery. Fractures that occurred 3 months after surgery were not included.

The average bone mineral density (BMD) was calcu- lated by averaging the values from L1 to L4. Segmental kyphotic angle was measured using Cobb's method from the superior endplate of the vertebra one level above the fractured vertebra to the inferior endplate of the vertebral body one level below the treated vertebra [13]. The vertebral body height ratio was calculated by comparing the height of the fractured segment to the mean height of the two adjacent segments.

\section{Surgical technique}

Indications for KP included recent fractures that were pain-resistant to analgesics for at least 3 weeks, as per a Visual Analog Scale (VAS) score of $>5$ points and a kyphotic deformity $>15^{\circ}$. Patients with neurological deficits, collapse rate $>90 \%$, unstable fractures, bleeding disorders, or any systemic or spinal infections were excluded.

Patients were asked to be in the prone position on the fluoroscopy table, and the procedures were performed under local anesthesia. Two 13-gauge long spinal needles were inserted into the pedicle and advanced to the anterior third of the vertebral body under C-arm fluoroscopic guidance. Thereafter, inflatable balloons were inserted into the vertebral body through two cannulas to create cavities. About 2-8 mL of PMMA bone cement, depending on the size of the vertebral body, were injected into these cavities. All the patients were fitted with rigid braces for 2-3 months after the procedure.

\section{Statistical analyses}

All the values in this paper are expressed in terms of mean \pm standard deviation, depending on the characteristics of the parameters. All data were subjected to statistical analyses using the Mann-Whitney $U$-test, the Student $t$-test, or the chi-square test. All $p<0.05$ were considered statistically significant. The statistical program IBM SPSS ver. 20.0 (IBM Corp., Armonk, NY, USA) was used.

This study was approved by the Institutional Review Boards of Inha University Hospital (IRB approval no., IUH-IRB 08-021). Informed consent was waived because of the retrospective nature of the study and the analysis used anonymous clinical data.

\section{Results}

In the 123 patients with painful OVCF, 143 vertebral bod- 
ies were treated with KP. Early adjacent vertebral fractures were present in $20(16.2 \%)$ of the 123 patients who underwent the KP, including in the cranial adjacent vertebra in 16 patients and caudal adjacent vertebra in four patients. The level of adjacent vertebral fracture was distributed between T10 and L3, with the most common vertebra being T12 (Fig. 1). Of the 123 patients, $110(89.4 \%)$ had a single vertebral fracture and $13(10.6 \%)$ had multiple vertebral fractures. The average follow-up was 6.8 months (range, 3-12.2 months), and the adjacent vertebral fracture was detected on an average 1.5 months (range, $0.8-3$ months) postoperatively.

There were no statistical differences with respect to sex, age, mean BMI, mean BMD, vertebral body height ratio, Cobb's angle, preoperative VAS score, and cement amount

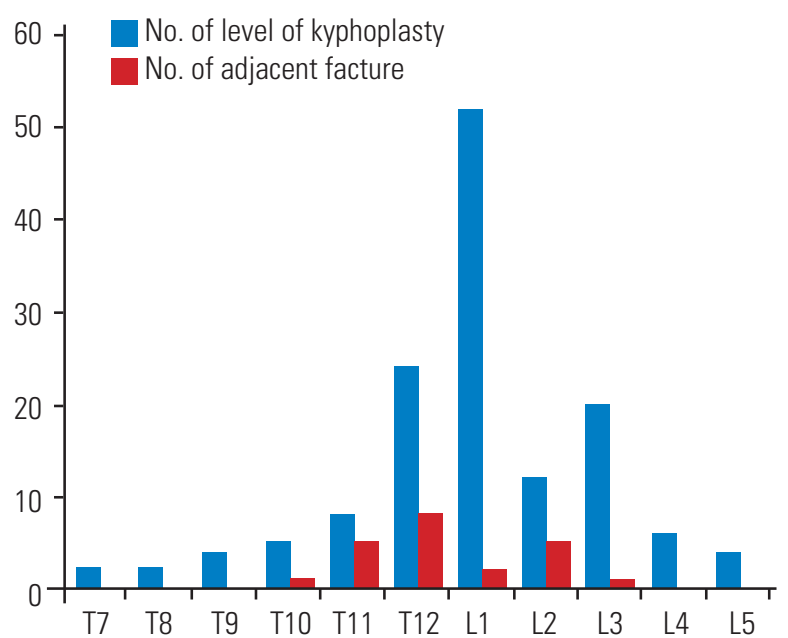

Fig. 1. Distribution of vertebral and adjacent fractures between the thoracic (T) and lumbar (L) vertebrae. between the fracture and control groups. The mean BMD was $3.5 \pm 1.19 \mathrm{~g} / \mathrm{m}^{2}$ in the fracture group and $3.72 \pm 1.25$ $\mathrm{g} / \mathrm{m}^{2}$ in the control group. The kyphotic angle was $22.02^{\circ} \pm 10.08^{\circ}$ in the fracture group and $24.61^{\circ} \pm 11.46^{\circ}$ in the control group. The mean volumes of the injected cement in the fracture control groups were $5.6 \pm 1.38 \mathrm{~mL}$ and $5.69 \pm 1.60 \mathrm{~mL}$, respectively.

The only statistically significant difference between the two groups was the cement leakage into the disc space (Table 1). Cement leakage into the disc space was identified in 16 of the 123 patients after surgery. Of these, 11 patients had adjacent vertebral fractures and five had cement leakage without adjacent vertebral fractures (Fig. 2). Cement leakage was present in significantly more patients (55\%) in the fracture group than in the control group (4.5\%). Cement leakage occurred above and below the fractured area, including cement leakage above the superior vertebral end plate in 11 patients, below the inferior vertebral end plate in four, and on both sides in one.

\section{Discussion}

In the literature, the incidence of adjacent vertebral fracture after balloon KP ranges from $6.5 \%$ to $25 \%$ [12,14-17]. Fribourg et al. [16] treated 38 patients at 47 levels with balloon KP; of these, 10 patients experienced 17 subsequent fractures. Harrop et al. [17] treated 225 vertebrae in 115 patients with balloon KP; 34 subsequent fractures developed in 26 patients within an average follow-up period of 1 month. In our study, the incidence of adjacent vertebral fracture after KP was $16.2 \%$, similar to that reported by earlier studies.

Table 1. Comparison of demographic and other characteristics of patients with and without adjacent vertebral fractures treated with balloon kyphoplasty

\begin{tabular}{lccc} 
Characteristic & Fracture group $(\mathrm{n}=20)$ & No fracture group $(\mathrm{n}=103)$ & $p$-value \\
\hline Sex (female:male) & $17: 3$ & $28: 75$ & 0.187 \\
\hline Age $(\mathrm{yr})$ & $78.0 \pm 3.8$ & $79.0 \pm 4.8$ & 0.321 \\
\hline Body mass index $\left(\mathrm{kg} / \mathrm{m}^{2}\right)$ & $23.10 \pm 3.8$ & $23.02 \pm 3.7$ & 0.188 \\
\hline Bone mineral density $\left(\mathrm{g} / \mathrm{m}^{2}\right)$ & $3.5 \pm 1.2$ & $3.7 \pm 1.3$ & 0.152 \\
\hline Vertebral body height ratio $(\%)$ & $52.04 \pm 14.32$ & $55.09 \pm 16.89$ & 0.096 \\
Cobb's angle $\left(^{\circ}\right)$ & $23.02 \pm 10.08$ & $24.61 \pm 11.46$ & 0.079 \\
\hline Preoperative Visual Analog Scale score & 8.5 & 8.8 & 0.820 \\
\hline Cement amount $(\mathrm{mL})$ & $5.6 \pm 1.38$ & $5.69 \pm 1.60$ & 0.321 \\
\hline Cement leakage & $11(55.0)$ & $5(4.5)$ & $<0.01$ \\
\hline
\end{tabular}

Values are presented as number, mean \pm standard deviation, or number (\%). 

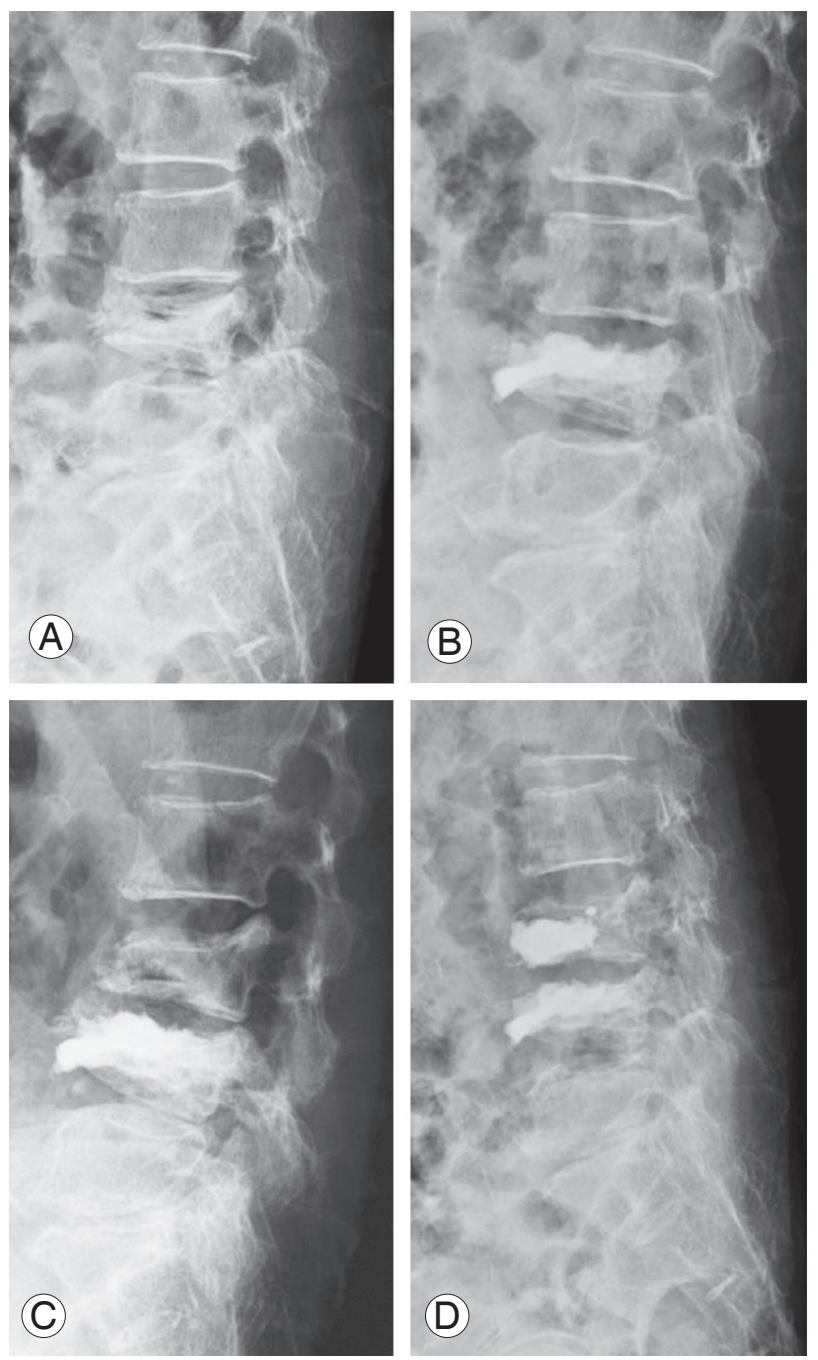

Fig. 2. An 87-year-old woman with a painful osteoporotic compression fracture. (A) Plain radiograph revealing an osteoporotic compression fracture at $L 4$. (B) Cement leakage into the L3-L4 disk after balloon kyphoplasty at L4. (C) Adjacent vertebral fracture at L3 that developed 3 months after balloon kyphoplasty. (D) The newly developed compression fracture at L3 was treated with balloon kyphoplasty.

It is unclear whether adjacent vertebral fractures develop as a natural progression of osteoporosis or as a consequence of stiffness because of the augmentation with bone cement. Rigid cement-augmented vertebra can be stiffer than the adjacent vertebrae. Baroud et al. [18] studied load shift of the intervertebral shift after vertebroplasty. That finite element study demonstrated that the cement in the vertebra reduced the physiologic inward bulge of the endplates and increased the pressure in the adjacent intervertebral disc by up to $17 \%$, possibly causing adjacent fractures [18].

Various risk factors for adjacent vertebral fractures af- ter cement augmentation have been reported, including quantitative CT-based BMD, bone cement distribution, greater volume of the injected cement, and cement leakage into the disc space [19-21]. Lin et al. [20] reported that $>50 \%$ of the adjacent vertebra with cement leakage into the disc space had fractures. Zhong et al. [21] also demonstrated that cement leakage was an independent risk factor for new fractures after KP. Cement leakage into the disc space may weaken the buffer capacity, a function of normal discs, resulting in more frequent adjacent vertebral fractures [21].

Our data demonstrated that cement leakage was a risk factor for adjacent vertebral fracture after KP. The present study identified a significant increase in the incidence of adjacent vertebral fractures in patients with cement leakage into the disk. It appears that cement leakage into the disk results in higher mechanical pressure, eventually causing injury to the endplate in the adjacent vertebral bodies.

In order to minimize cement leakage into the disk, proper surgical techniques are necessary, including correct balloon placement, high-viscosity PMMA cement, controlled application of the cement into the vertebra, and limiting the injected cement volume. Greene et al. [13] suggested the use of the eggshell-technique to prevent further cement leakage, where after primary reduction with the balloon, a small amount of doughy cement is applied into the cavity; therefore, the balloon is re-inflated. Cortical defects may be closely associated with the risk of cement leakage. In cases with cortical defects, highviscosity bone cement is preferable. If leakage is suspected in the fluoroscopic image during the procedure, the injection should be stopped temporarily, allowing a check for cement leakage into the disc space or the paraspinal space. Waiting a few minutes can enhance the cement viscosity and reduce leakage through the defect.

It remains debatable whether the volume of injected cement is a risk factor for adjacent vertebral fracture. Theoretically, a large volume of the injected cement may increase the chances of cement leakage and may be associated with increased mechanical pressure on the adjacent vertebral bodies. In contrast, some studies have reported that the volume of the cement was not a risk factor because an optimal volume of bone cement has not been determined thus far and can change depending on the vertebral body size [22]. A bigger vertebral body requires a greater cement volume to fill the body. In our study, the 
volume of the injected cement was not a risk factor for adjacent fracture.

Most adjacent vertebral fractures occur within 3 months of KP. KP decreases back pain postoperatively, increasing the patient's ability to perform daily activities and leading to a higher chance of increased mechanical pressure on the endplate of the adjacent vertebral bodies. One strategy to reduce the risk of early adjacent segment fracture is the application of a rigid brace for few months after the surgery in case of cement leakage.

This study has certain limitations. First, we enrolled a relatively small number of patients. Therefore, a consecutive study is required. Second, although several factors affect adjacent vertebral fracture after KP, we were unable to investigate whether patients were on medication for osteoporosis before the procedure. Thus, the effect of osteoporosis medication was not considered in the present study.

\section{Conclusions}

Balloon KP is a minimally invasive procedure for stabilizing vertebral compression fractures and is effective in pain reduction. However, early adjacent vertebral fractures were present in $16 \%$ of the patients within 3 months after surgery. The most important risk factor for fractures was cement leakage into the disk. Thus, careful attention should be paid to determine whether cement leakage occurs during KP.

\section{Conflict of Interest}

No potential conflict of interest relevant to this article was reported.

\section{Author Contributions}

Cho KJ: corresponding author, conception and design, critically revision, and supervision; and Ko BS, Park JW: drafting of the manuscript, acquisition of data, and analysis.

\section{References}

1. Johnell O, Kanis JA. An estimate of the worldwide prevalence and disability associated with osteoporotic fractures. Osteoporos Int 2006;17:1726-33.
2. Borgstrom F, Zethraeus N, Johnell O, et al. Costs and quality of life associated with osteoporosis-related fractures in Sweden. Osteoporos Int 2006;17:637-50.

3. Ensrud KE, Thompson DE, Cauley JA, et al. Prevalent vertebral deformities predict mortality and hospitalization in older women with low bone mass. Fracture Intervention Trial Research Group. J Am Geriatr Soc 2000;48:241-9.

4. Garfin SR, Yuan HA, Reiley MA. New technologies in spine: kyphoplasty and vertebroplasty for the treatment of painful osteoporotic compression fractures. Spine (Phila Pa 1976) 2001;26:1511-5.

5. Garfin SR, Buckley RA, Ledlie J; Balloon Kyphoplasty Outcomes Group. Balloon kyphoplasty for symptomatic vertebral body compression fractures results in rapid, significant, and sustained improvements in back pain, function, and quality of life for elderly patients. Spine (Phila Pa 1976) 2006;31:2213-20.

6. Grafe IA, Da Fonseca K, Hillmeier J, et al. Reduction of pain and fracture incidence after kyphoplasty: 1-year outcomes of a prospective controlled trial of patients with primary osteoporosis. Osteoporos Int 2005;16:2005-12.

7. Komp M, Ruetten S, Godolias G. Minimally-invasive therapy for functionally unstable osteoporotic vertebral fractures by means of kyphoplasty: prospective comparative study of 19 surgically and 17 conservatively treated patients. J Miner Stoffwechs 2004;11:604-12.

8. Bouza C, Lopez T, Magro A, Navalpotro L, Amate JM. Efficacy and safety of balloon kyphoplasty in the treatment of vertebral compression fractures: a systematic review. Eur Spine J 2006;15:1050-67.

9. Campbell PG, Harrop JS. Incidence of fracture in adjacent levels in patients treated with balloon kyphoplasty: a review of the literature. Curr Rev Musculoskelet Med 2008;1:61-4.

10. Robinson Y, Tschoke SK, Stahel PF, Kayser R, Heyde CE. Complications and safety aspects of kyphoplasty for osteoporotic vertebral fractures: a prospective follow-up study in 102 consecutive patients. Patient Saf Surg 2008;2:2.

11. Spross C, Aghayev E, Kocher R, Roder C, Forster T, Kuelling FA. Incidence and risk factors for early adjacent vertebral fractures after balloon kyphoplasty for osteoporotic fractures: analysis of the SWISSspine registry. Eur Spine J 2014;23:1332-8. 
12. Lin EP, Ekholm S, Hiwatashi A, Westesson PL. Vertebroplasty: cement leakage into the disc increases the risk of new fracture of adjacent vertebral body. AJNR Am J Neuroradiol 2004;25:175-80.

13. Greene DL, Isaac R, Neuwirth M, Bitan FD. The eggshell technique for prevention of cement leakage during kyphoplasty. J Spinal Disord Tech 2007;20:22932.

14. Frankel BM, Monroe T, Wang C. Percutaneous vertebral augmentation: an elevation in adjacent-level fracture risk in kyphoplasty as compared with vertebroplasty. Spine J 2007;7:575-82.

15. Movrin I, Vengust R, Komadina R. Adjacent vertebral fractures after percutaneous vertebral augmentation of osteoporotic vertebral compression fracture: a comparison of balloon kyphoplasty and vertebroplasty. Arch Orthop Trauma Surg 2010;130:1157-66.

16. Fribourg D, Tang C, Sra P, Delamarter R, Bae H. Incidence of subsequent vertebral fracture after kyphoplasty. Spine (Phila Pa 1976) 2004;29:2270-6.

17. Harrop JS, Prpa B, Reinhardt MK, Lieberman I. Primary and secondary osteoporosis' incidence of subsequent vertebral compression fractures after kyphoplasty. Spine (Phila Pa 1976) 2004;29:2120-5.

18. Baroud G, Nemes J, Heini P, Steffen T. Load shift of the intervertebral disc after a vertebroplasty: a finiteelement study. Eur Spine J 2003;12:421-6.

19. Lo YP, Chen WJ, Chen LH, Lai PL. New vertebral fracture after vertebroplasty. J Trauma 2008;65:143945.

20. Lin J, Qian L, Jiang C, Chen X, Feng F, Lao L. Bone cement distribution is a potential predictor to the reconstructive effects of unilateral percutaneous kyphoplasty in OVCFs: a retrospective study. J Orthop Surg Res 2018;13:140.

21. Zhong BY, He SC, Zhu HD, et al. Risk prediction of new adjacent vertebral fractures after PVP for patients with vertebral compression fractures: development of a prediction model. Cardiovasc Intervent Radiol 2017;40:277-84.

22. Kim DJ, Kim TW, Park KH, Chi MP, Kim JO. The proper volume and distribution of cement augmentation on percutaneous vertebroplasty. J Korean Neurosurg Soc 2010;48:125-8. 